\title{
Unification of the Genera Deleya (Baumann et al. 1983), Halomonas (Vreeland et al. 1980), and Halovibrio (Fendrich 1988) and the Species Paracoccus halodenitrificans (Robinson and Gibbons 1952) into a Single Genus, Halomonas, and Placement of the Genus Zymobacter in the Family Halomonadaceae
}

\author{
S. J. DOBSON* AND P. D. FRANZMANN $\dagger$ \\ Antarctic Cooperative Research Centre and Department of Agricultural Science, \\ University of Tasmania, Hobart, Tasmania 7001, Australia
}

\begin{abstract}
We determined the $16 \mathrm{~S}$ rRNA sequences of the type strains of species belonging to the genera Deleya and Halomonas for which no sequence data were available previously. We also determined the 16S rRNA sequence of ACAM 21, a representative strain of a biovar of Halomonas subglaciescola. The members of the genera Deleya, Halomonas, and Halovibrio and the misnamed organism Paracoccus halodenitrificans formed a monophyletic group within the gamma subclass of the Proteobacteria. The 16S rRNA sequences of the members of this group contained all of the signature features previously identified as characteristic of the group. The frequency of occurrence of these signature features among other members of the gamma subclass has remained stable during the expansion of the database of rRNA sequences. The levels of $16 \mathrm{~S}$ rRNA sequence similarity between members of the species belonging to the genera Deleya, Halomonas, and Halovibrio and the misnamed organism $P$. halodenitrificans ranged from 91.5 to $100 \%$; however, the level of sequence similarity for members of well-resolved monophyletic subgroups which might represent separate genera was $98 \%$. At a sequence similarity level of $98 \% 10$ subgroups were resolved, but these groups could not be differentiated on the basis of chemotaxonomic or phenotypic characteristics. In this paper we propose that members of the genera Deleya, Halomonas, and Halovibrio should be placed in a single genus, the genus Halomonas, and we emend the description of this genus. The resulting new combinations are Halomonas aquamarina (Deleya aquamarina Akagawa and Yamasato 1989), Halomonas variabilis (Halovibrio variabilis Fendrich 1988), Halomonas venusta (Deleya venusta Baumann et al. 1983), Halomonas cupida (Deleya cupida Baumann et al. 1983), Halomonas pacifica (Deleya pacifica Baumann et al. 1983), Halomonas marina (Deleya marina Baumann et al. 1983), Halomonas halophila (Deleya halophila Quesada et al. 1984), and Halomonas salina (Deleya salina Valderrama et al. 1991). We transfer the misnamed organism $P$. halodenitrificans to the genus Halomonas as Halomonas halodenitrificans comb. nov. (P. halodenitrificans Robinson and Gibbons 1952). The genus Zymobacter is closely related to the genus Halomonas. While the genus Zymobacter can be clearly distinguished from the genus Halomonas, these two taxa share important genotypic, chemotaxonomic, and phenotypic characteristics. We propose that the genus Zymobacter should be transferred to the family Halomonadaceae and emend the description of the family Halomonadaceae. The 16S rRNA sequence of Halomonas subglaciescola ACAM 21 was significantly different from the 16S rRNA sequence of the type strain of Halomonas subglaciescola (strain ACAM 12) but was nearly identical to the 16S rRNA sequence of Halomonas halodurans.
\end{abstract}

Species belonging to the genera Deleya, Halomonas, and Halovibrio are heterotrophic, gram-negative, rod-shaped bacteria which have been isolated from seawater, estuarine water, hypersaline soils, and bodies of hypersaline water, including Antarctic lakes and the Dead Sea. These species are aerobic, and some strains have the capacity for facultative anaerobic growth in the presence of nitrate. Members of each genus grow over a wide range of levels of salinity. All of the species require salt for optimal growth and are therefore classified as halophiles according to the definition of Larsen (17). The level of salt required for optimal growth varies among species. For

\footnotetext{
* Corresponding author. Mailing address: Antarctic CRC and Department of Agricultural Science, University of Tasmania, GPO Box 252C, Hobart, Tasmania 7001, Australia. Electronic mail address: sue .dobson@agsci.utas.edu.au.

$\dagger$ Present address: CSIRO Division of Water Resources, Floreat Park, Western Australia 6014, Australia.
}

example, Deleya halophila grows optimally in media containing $7.5 \%$ marine salts, Deleya salina grows optimally in media containing $5 \%$ salts, and Halomonas meridiana grows optimally in media containing 1 to $3 \% \mathrm{NaCl}(13,24,31)$.

The family Halomonadaceae was created to accomodate the genera Deleya and Halomonas (11). These two genera have similar fatty acid, polar lipid, and respiratory lipoquinone profiles (10). Chemotaxonomic data indicated that the genus Halovibrio, which contains a single species, was related to the genera Deleya and Halomonas, and this was confirmed by the results of a comparison of 16S rRNA sequences (5). An analysis of the 16S rRNA sequences of a representative group of eight species demonstrated that the members of the genera Deleya and Halomonas do not form monophyletic groups corresponding to their generic assignments (5). Members of all three genera had several signature characteristics in their $16 \mathrm{~S}$ rRNA sequences which were common to all of them and which defined this group of bacteria within the gamma subclass of the 
Proteobacteria. The current arrangement of species in the genera Deleya and Halomonas has no taxonomic validity.

Well-resolved groups which might form the basis of genera could not be identified on a tree derived from the $16 \mathrm{~S}$ rRNA data. When the formation of genera was considered, the chemotaxonomic differences were too small to describe separate genera. Phenotypic characteristics which defined groups at the generic level could not be identified. As several species belonging to the genera Deleya and Halomonas were not included in this 16S rRNA study, a formal proposal for unification of these three genera was considered premature. In this study we determined the 16S rRNA sequences of the remaining species belonging to the genera Deleya and Halomonas. Mellado et al. (18) recently published the $16 \mathrm{~S}$ rRNA sequences of two strains included in this study, D. salina ATCC $495509^{\mathrm{T}}(\mathrm{T}=$ type strain) and Volcaniella eurihalina ATCC $49336^{\mathrm{T}}$, and proposed that $V$. eurihalina should be transferred to the genus Halomonas. Our results and the results of Mellado et al. are compared below.

An analysis of the 16S rRNA sequence of Paracoccus halodenitrificans revealed that this species belongs in the family Halomonadaceae. However, the lack of meaningful taxonomic structure within the Halomonadaceae at the genus level made assignment of a genus name to the misnamed organism Paracoccus halodenitrificans an arbitrary and artificial exercise (19). Until the genera in this family are redefined, misnamed and newly described species will remain in a state of limbo.

Strain ACAM 21 is a representative of a biovar of Halomonas subglaciescola (9). The members of this biovar were distinguished from the members of the biovar containing type strain ACAM 12 by a lack of motility, a lack of peritrichous flagella, and a cell length of less than $10 \mu \mathrm{m}$. Other phenotypic differences between the two biovars included differences in utilization of asparagine, surface growth in thioglycolate broth, and susceptibility to penicillin $\mathrm{G}$. There are significant differences in the proportions of fatty acids in strain ACAM 21 and the type strain of Halomonas subglaciescola strain ACAM 12. The levels of the fatty acids $16: 0,16: 1$ plus $17: 0 \mathrm{cyc}$, and 18:1 plus 19:0cyc were $25.4,19.7$, and $52.3 \%$ (wt/wt) and 32.0, 28.9, and $28.2 \%$ (wt/wt) for strains ACAM $12^{\mathrm{T}}$ and ACAM 21, respectively, when the organisms were grown under identical conditions (10). The 16S rRNA sequence of strain ACAM 21 was determined to test the hypothesis that the biovar that contains this strain represents a species that is distinct from Halomonas subglaciescola.

\section{MATERIALS AND METHODS}

Microorganisms. Halomonas halodurans DSM $5160^{\mathrm{T}}$ was obtained from the Deutsche Sammlung von Mikroorganismen und Zellkulturen GmbH, Braunschweig, Germany. Halomonas subglaciescola ACAM 21, Halomonas eurihalina ACAM $353^{\mathrm{T}}\left(=\right.$ ATCC $\left.49336^{\mathrm{T}}\right)$, Deleya venusta ACAM $346^{\mathrm{T}}\left(=\operatorname{DSM} 4743^{\mathrm{T}}\right)$, Deleya pacifica ACAM $345^{\mathrm{T}}$ (= DSM $4742^{\mathrm{T}}$ ), and Deleya cupida ACAM $343^{\mathrm{T}}$ $\left(=\operatorname{DSM} 4740^{\mathrm{T}}\right.$ ) were obtained from the Australian Collection of Antarctic Microorganisms, University of Tasmania, Hobart, Tasmania, Australia. D. salina ATCC $49509^{\mathrm{T}}$ was kindly supplied by A. Ventosa.

Halomonas subglaciescola ACAM 21 was grown in artificial organic lake water peptone broth (9). The other strains were grown in a marine broth supplemented with glucose $(0.5 \%$ [wt/vol] peptone, $0.1 \%$ [wt/vol] yeast extract, and $1.0 \%$ [wt/vol] glucose in seawater that had been filtered [pore size, $0.45 \mu \mathrm{m}$ ]). All strains were grown at $25^{\circ} \mathrm{C}$.

Determination of $16 \mathrm{~S}$ rRNA sequences. The methods used to extract DNAs from the strains and to amplify the 16S rRNA gene by the PCR have been described previously (4). The sequence data for Halomonas halodurans, Halomonas subglaciescola ACAM 21, D. verusta, and D. salina were obtained by directly sequencing the isolated $16 \mathrm{~S}$ ribosomal DNA PCR products. Most of these sequence data were obtained by manual sequencing, as described previously (4). A small amount of the sequence data for these strains was obtained by directly sequencing the isolated $16 \mathrm{~S}$ ribosomal DNA PCR products with a PRISM Ready Reaction dideoxy termination cycle sequencing kit (Perkin Elmer); for electro- phoresis and data collection we used an Applied Biosystems model 373A DNA sequencer (Perkin Elmer). The $16 S$ ribosomal DNA PCR products of $D$. pacifica, D. cupida, and Halomonas eurihalina which we isolated were cloned by using the pGEM-T vector system (Promega). Plasmid DNA was isolated by using the Wizard DNA purification system (Promega). A sample of an isolated plasmid was digested with restriction enzyme PvuII (Promega), and the products of this digestion were electrophoresed in an agarose minigel (27) to make sure that the cloned insertion was the correct size. Plasmids containing a fragment of the correct size were sequenced by using a PRISM Ready Reaction dideoxy termination cycle sequencing kit (Perkin Elmer) and an Applied Biosystems model 373A DNA sequencer (Perkin Elmer) for electrophoresis and data collection.

$16 S$ rRNA data analysis. The $16 \mathrm{~S}$ rRNA sequences which we obtained were manually aligned with previously determined sequences of members of the Halomonadaceae and some members of the gamma subclass of the Proteobacteria, including the nearest relatives of the Halomonadaceae. The strains and GenBank accession numbers for the previously determined 16S rRNA sequences are as follows (for sequences which are not present in the GenBank database the Ribosomal Database Project code is given): Paracococcus halodenitrificans DSM $735^{\mathrm{T}}$, L04942; Halomonas halmophila ATCC $19717^{\mathrm{T}}$, M59153; Halomonas elongata DSM 2851 ${ }^{\mathrm{T}}$, M93355; Halomonas subglaciescola ACAM 12 ${ }^{\mathrm{T}}, \mathrm{M} 93358$; Halomonas meridiana ACAM 246 ${ }^{\mathrm{T}}$, M93356; D. halophila DSM 4770 ${ }^{\mathrm{T}}$, M93353; Deleya aquamarina DSM $30161^{\mathrm{T}}, \mathrm{M} 93352$; Deleya marina DSM $4741^{\mathrm{T}}, \mathrm{M}^{2} 3354$; Halovibrio variabilis DSM $3051^{\mathrm{T}}$, M93357; Chromohalobacter marismortui ATCC $17056^{\mathrm{T}}$, X87219; C. marismortui str. A-65, X87220; Zymobacter palmae IAM $14233^{\mathrm{T}}$, D14555; Oceanospirillum minutulum ATCC $19193^{\mathrm{T}}$, osp.minutu; Oceanospirillum linum ATCC $11336^{\mathrm{T}}$, M22365; Marinomonas vaga ATCC $27119^{\mathrm{T}}$, mrm. vaga; Pseudomonas aeruginosa ATCC 25330, M34133; Pseudomonas nautica IAM 12929, D11189, D11238, and D11287; and Alteromonas haloplanktis ATCC $14393^{\mathrm{T}}$, X67024. Most of the 16S rRNA sequences of the reference species and the previously determined sequences of members of the Halomonadaceae were obtained from the Ribosomal Database Project (22); the exceptions were the sequences of $C$. marismortui ATCC $17056^{\mathrm{T}}$ and str. A-65, which were obtained from GenBank. Almost-complete sequences were available for all of the reference species except $P$ seudomonas nautica, for which only a partial sequence (440 baes) was available. Consequently, Pseudomonas nautica was not included in most analyses.

The sequences which we determined were examined for the presence of the 19 signature characteristics of the Halomonadaceae (5). Trees were constructed by using distance and parsimony methods. Programs in the PHYLIP version 3.4 package were used for the distance analysis (6). Jukes-Cantor evolutionary distances were calculated by using the DNADIST program, and a tree was constructed from these distances by using the FITCH program and the global rearrangement option. PAUP version 3.1 was used for the parsimony analysis (29). Most-parsimonious trees were obtained by the branch-and-bound method. Levels of similarity between sequences were determined from the mean distances calculated with PAUP version 3.1. A bootstrap analysis (2,000 replications) was performed for both the distance and parsimony methods. The parsimony bootstrap analysis was performed by using a heuristic search method because of computing limitations.

The database of $16 \mathrm{~S}$ rRNA sequences continues to expand rapidly (22). A total of $3816 \mathrm{~S}$ rRNA sequences of organisms belonging to the gamma subclass of the Proteobacteria were present in the database when the 19 signature characteristics of the members of the Halomonadaceae were identified (5). The ribosomal database now contains 433 6S rRNA sequences of organisms belonging to this subclass. The 395 new sequences were examined for the presence of the signature characteristics of the Halomonadaceae to determine whether the rare occurrence of the full set of signature characteristics observed previously has been maintained. All of the 16S rRNA sequences in the database were examined to determine whether they contained the rarest of the signature characteristics, a cytosine residue at position 486 (Escherichia coli numbering).

Nucleotide sequence accession numbers. The sequence data determined in this study have been deposited in the GenBank database under the following accession numbers: Halomonas subglaciescola ACAM 21, L42614; D. cupida DSM 4740 ${ }^{\mathrm{T}}, \mathrm{L} 42615$; D. pacifica DSM $4742^{\mathrm{T}}$, L42616; D. salina ATCC $49509^{\mathrm{T}}$, L42617; D. venusta DSM 4743 ${ }^{\mathrm{T}}$, L42618; Halomonas halodurans DSM $5160^{\mathrm{T}}$, L42619; and $V$. eurihalina ATCC $49336^{\mathrm{T}}$, L42620.

\section{RESULTS}

The amounts of the $16 \mathrm{~S}$ rRNA gene sequence determined for the seven strains which we examined ranged from 91.8 to $99.1 \%$ (average, 96.5\%) in the region from position 29 to position 1521 ( $E$. coli numbering). The $16 \mathrm{~S}$ rRNA sequences of $D$. cupida, D. pacifica, D. venusta, Halomonas halodurans, and Halomonas subglaciescola ACAM 21 all contained the 19 signature features previously identified as characteristic features of the family Halomonadaceae in the gamma subclass of the Proteobacteria. Table 1 shows the levels of similarity for mem- 


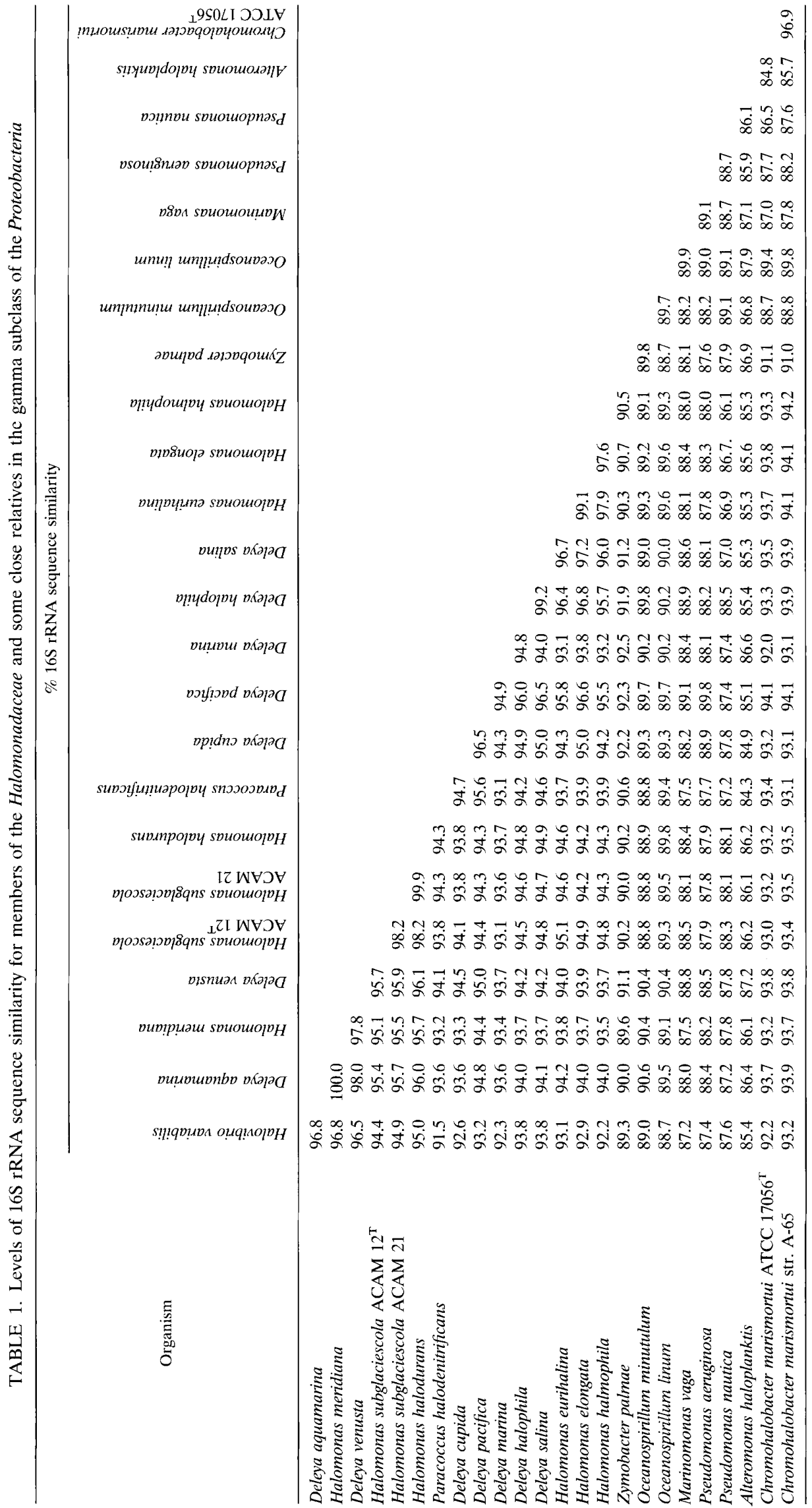


bers of the Halomonadaceae and the nearest relatives of this family.

The level of similarity of the $16 \mathrm{~S}$ rRNA sequences of Halomonas eurihalina ATCC $49336^{\mathrm{T}}$ determined by Mellado et al. (18) and in this study was $99.5 \%$. The $0.5 \%$ difference between the two sequences represents experimental error or microheterogeneity in the $16 \mathrm{~S}$ rRNA gene, which may be present in more than one copy in the genome, or a combination of both of these factors. The level of similarity of the $16 \mathrm{~S}$ rDNA sequences of $D$. salina ATCC $49509^{\mathrm{T}}$ determined by Mellado et al. (18) and in this study was $96.3 \%$, which was much lower than the value that might be expected as a result of experimental error. A comparison of primary and secondary structural features did not indicate substantial errors in either sequence. At most points at which the sequences varied in helical sections of the secondary structure, there was a concomitant variation in the paired bases of the helix to maintain canonical base pairing. Where canonical base pairing was not maintained or where the variation occurred in a loop section, both sequence variations also occurred in other species belonging to the Halomonadaceae, with a few exceptions. These exceptions were a deletion at position 98 , a $\mathrm{G}$ at position 1243 , and $\mathrm{a} G$ at position 1286 ( $E$. coli numbering) which occurred in the sequence of Mellado et al. (18) but were not present in the sequence of any other species belonging to the Halomonadaceae. The low level of similarity between the 16S rRNA sequences determined by Mellado et al. and by us suggests that two different strains were sequenced. Both strains belonged to the Halomonadaceae. The strain used in this study was closely related to $D$. halophila (level of $16 \mathrm{~S}$ rRNA sequence similarity, $99.2 \%$ ), whereas the strain used in the study of Mellado et al. (18) was equidistant from Halomonas elongata, D. aquamarina, and $D$. halophila (levels of $16 \mathrm{~S}$ rRNA sequence similarity, 96.3, 96.2 , and $96.7 \%$, respectively). Further investigation is required to confirm which strain is the authentic type strain.

There are other differences between our 16S rRNA similarity results and those of Mellado et al. (18). The 16S rRNA similarity values obtained for Halomonas elongata, Halomonas meridiana, Halomonas subglaciescola, D. aquamarina, D. halophila, and Halovibrio variabilis in the two studies are similar, but the values obtained for D. marina, and Halomonas halmophila are significantly different. The levels of 16S rRNA similarity between Halomonas halophila and the six species listed above were 1.6 to $1.9 \%$ higher in our study than in the study of Mellado et al. (18). When $D$. marina was studied, the levels of 16S rRNA similarity betwen this species and four of the species listed above were 3.3 to $3.8 \%$ higher in this study than in the study of Mellado et al. (18) and the levels of $16 \mathrm{~S}$ rRNA similarity between this species and the other two species were 0.8 to $1.3 \%$ lower. As the sequences which were used in the two studies were the same, the differences must have arisen from differences in the sequence alignments. All of the values given in this paper are values which were generated in this study.

The trees obtained by the distance and parsimony methods are shown in Fig. 1 and 2, respectively. D. cupida, D. pacifica, D. venusta, and Halomonas halodurans fell within the Halomonadaceae. $D$. venusta was closely related to $D$. aquamarina, which is consistent with DNA-rRNA hybridization data (15). Halomonas halodurans and strain ACAM 21 had almost identical sequences.

There were two equally most-parsimonious trees which varied only in the internal arrangement of the Halomonas eurihalina-Halomonas elongata-Halomonas halmophila cluster. The branching structures of the distance and parsimony trees were similar; however, several of the groups of species oc- curred in less than $90 \%$ of the bootstrap consensus trees obtained with the two methods (Fig. 3). This indicated that there was some instability in the arrangement of the species. Halomonas meridiana, Halomonas halodurans, and D. salina were not included in the bootstrap analyses described in this paper since in previous analyses these species grouped with $D$. aquamarina, strain ACAM 21, and D. salina, respectively, in $100 \%$ of the bootstrap trees, and we believed that excluding them would not significantly alter the results. However, their exclusion did affect the result. In the previous bootstrap parsimony analyses the group containing Halovibrio variabilis, $D$. aquamarina, Halomonas meridiana, D. venusta, Halomonas subglaciescola ACAM 12 ${ }^{\mathrm{T}}$, strain ACAM21, and Halomonas halodurans appeared less frequently, occurring in $66 \%$ of the bootstrap trees (not shown).

The frequency of the signature characteristics of members of the Halomonadaceae in the 433 16S rRNA sequences of members of the gamma subclass of the Proteobacteria currently available was similar to the frequency which occurred in the 38 sequences available 3 years ago (Table 2). Four of the signature characteristics are relatively common and occur in 18 to $24 \%$ of $16 \mathrm{~S}$ rRNA sequences of members of the gamma subclass; however, only $4.6 \%$ of the sequences contained 4 of the 19 signature characteristics, illustrating that in combination the signature characteristics provide an extremely effective way to describe the Halomonadaceae (Table 3). Only 3.8\% of the sequences contained between 7 and 10 of the signature characteristics, and there was only one species, Z. palmae, which contained more than 10 (this species contained 15 of the signature characteristics).

The rarest signature characteristic is a cytosine residue at position 486. When all available sequences were examined 3 years ago, a $\mathrm{C}$ occurred at position 486 in only one other prokaryote, Listonella anguillarum (5). An examination of all prokaryotic sequences currently available revealed a $\mathrm{C}$ at position 486 in only the following six sequences: Listonella anguillarum, Legionella hackeliae, Z. palmae, Isosphaera pallida, Magnetobacterium bavaricum, and Staphylococcus schleiferi subsp. schleiferi sequences. The last three species do not belong to the gamma subclass of the Proteobacteria. The $16 \mathrm{~S}$ rRNA sequence of Legionella hackeliae contains only two other signature characteristics associated with the members of the Halomonadaceae, and the 16S rRNA sequence of Listonella anguillarum contains none of the other signature characteristics, although only a partial sequence is available for this species. Z palmae is the only one of the six species which exhibits a significant level of similarity to members of the Halomonadaceae in terms of signature characteristics.

\section{DISCUSSION}

The genus Halomonas was described to accommodate a group of moderately halophilic strains isolated from a saltern (33). In the initial study of the strains assigned to the genus Halomonas, the researchers did not compare them with the marine Alcaligenes strains which later formed the genus Deleya. Similarly, in the studies which were the basis of the description of the genus Deleya the researchers did not compare the Deleya strains with Halomonas elongata which at that time was the sole species in the genus Halomonas (3). Following the initial descriptions of these genera, additional new species were assigned to them, primarily on the basis of phenotypic characteristics and DNA $\mathrm{G}+\mathrm{C}$ contents. Creation of the genus Halovibrio was based on phenotypic distinction from previously described genera, including the genus Halomonas (7).

The species belonging to the genera Deleya, Halomonas, and 


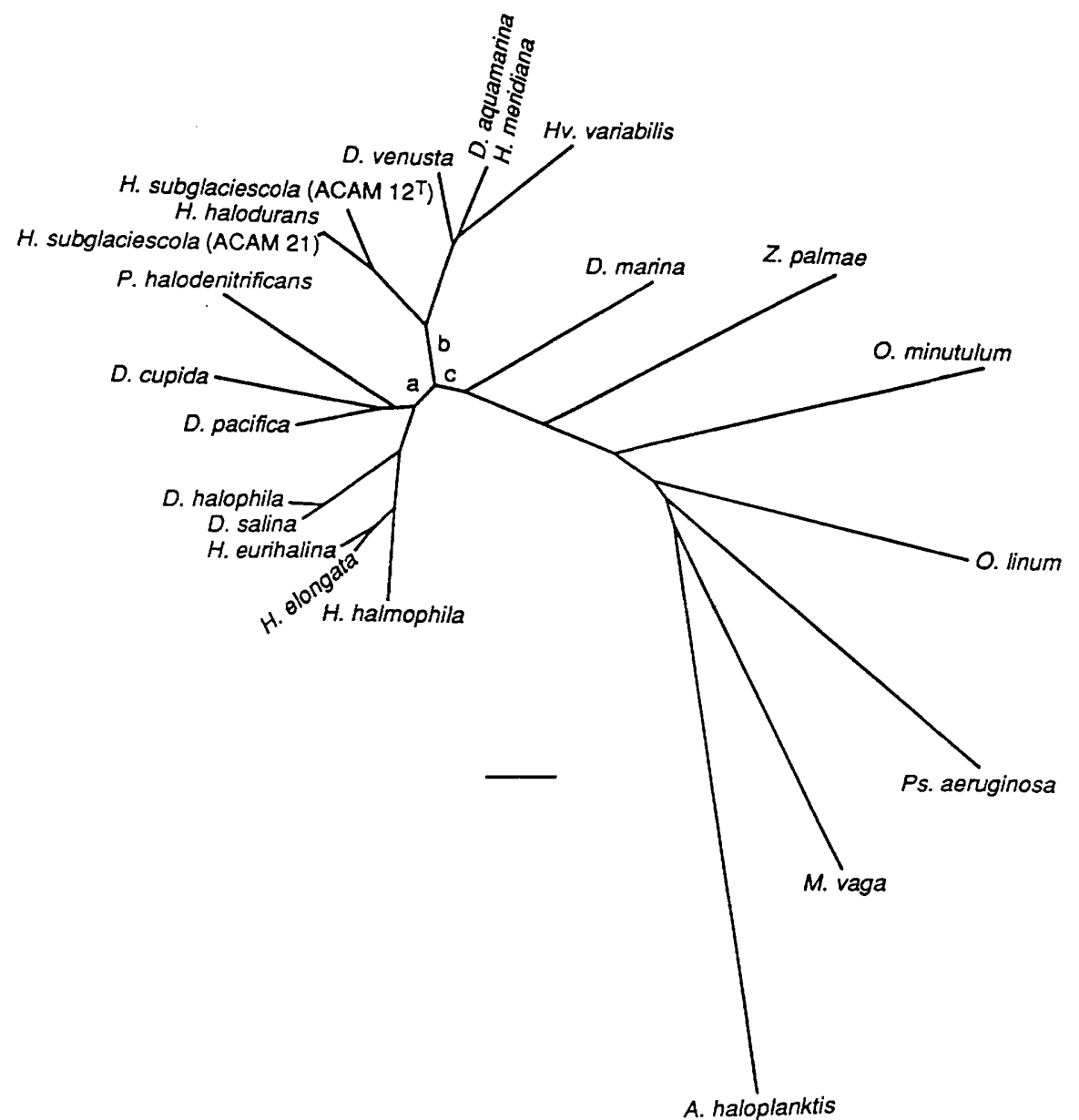

FIG. 1. Distance tree containing the members of the genera Deleya, Halomonas, and Halovibrio, the misnamed organism Paracoccus halodenitrificans, and some members of the gamma subclass of the Proteobacteria. Bar $=0.01$ distance calculated by the Jukes-Cantor equation. $a, b$, and $c$ are short internodes connecting the main branches of the monophyletic group containing the members of the genera Deleya, Halomonas, and Halovibrio. Abbreviations: $D$., Deleya; H., Halomonas; Hv., Halovibrio; Z., Zymobacter; O., Oceanospirillum; Ps., Pseudomonas; M., Marinomonas; A., Alteromonas; P., Paracoccus.

Halovibrio and the misclassified organism Paracoccus halodenitrificans form a coherent monophyletic group in trees derived from 16S rRNA sequence comparisons. This is evident not only from the levels of similarity obtained for the members of this group when they are compared with their nearest relatives, $Z$. palmae and $O$. minutulum, but also from the extensive set of signature characteristics associated with this group of species.

We needed to consider whether there are monophyletic subgroups within the set of species belonging to the genera Deleya, Halomonas, and Halovibrio and Paracoccus halodenitrificans which might deserve recognition as separate genera. The description of a genus requires not only that a group of species be phylogenetically coherent as inferred from genotypic data but that there be chemotaxonomic or phenotypic features characteristic of the group which differentiate it from closely related genera. As the Ad Hoc Committee on Approaches to Taxonomy within the Proteobacteria states, "The first step in the identification of bacteria is the assignment of organisms to genera. It is completely impracticable to define genera solely on the basis of phylogenetic data" (20).

There is a level of DNA-DNA hybridization $(70 \%)$ which is widely accepted as a basis for defining bacterial species (34), but no level of 16S rRNA sequence similarity has been arrived at for defining bacterial genera. This is because the ages of genera over the full breadth of evolutionary history are different and a single figure is not relevant for such a diverse range of organisms (28). It is also because of the practical requirement for data other than genotypic data to define genera. When the species belonging to the Halomonadaceae on the distance tree (Fig. 1) were examined, 10 distinct groups formed at a level of $16 \mathrm{~S}$ rRNA similarity of $98.0 \%$. Five of these groups each contained a single species. The groups contained the following organisms: group 1, Halovibrio variabilis; group 2, D. aquamarina, Halomonas meridiana, and D. venusta; group 3 , Halomonas subglaciescola, strain ACAM 21, and Halomonas halodurans; group 4, Paracoccus halodenitrificans; group 5, D. cupida; group 6, D. pacifica; group 7,D. marina; group 8, D. halophila and D. salina; group 9, Halomonas eurihalina and Halomonas elongata; and group 10, Halomonas halophila. At a level of similarity of $97 \%$ the separation of some species into distinct groups is not clear-cut. Groups 8, 9, and 10 begin to merge as the level of similarity between Halomonas elongata and $D$. salina is $97.2 \%$; however, the levels of similarity among this group of five species are as low as $95.7 \%$. In addition, the level of similarity between D. aquamarina and Halovibrio variabilis is $96.8 \%$. It is unlikely that a difference of $0.2 \%$ is meaningful simply because a value falls below the $97.0 \%$ level. At lower levels of similarity the formation of distinct groups be- 


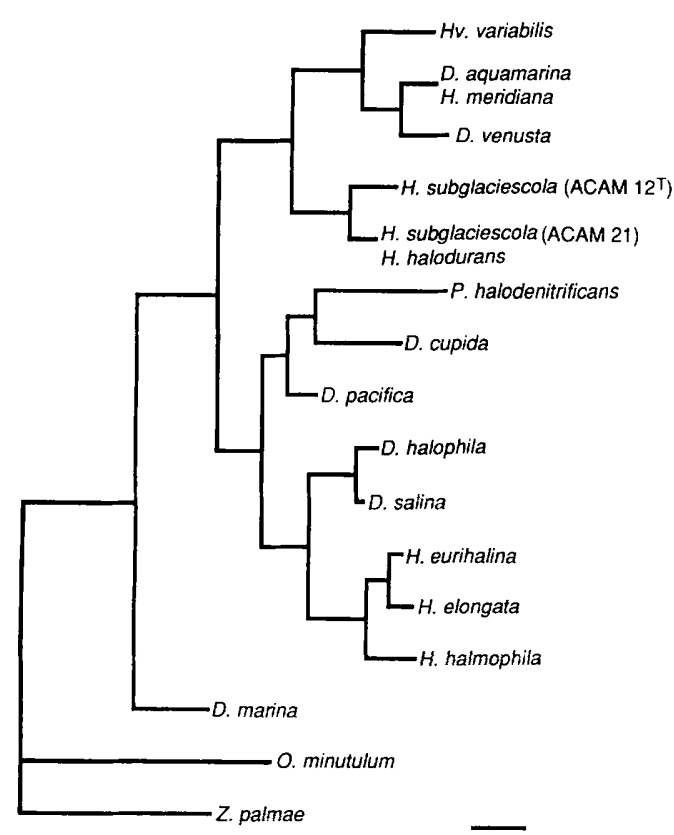

FIG. 2. Most-parsimonious tree containing the members of the genera $D e$ leya, Halomonas, and Halovibrio, the misnamed organism Paracoccus halodenitrificans, and some members of the gamma subclass of the Proteobacteria. Bar = 50 nucleotides. A second equally most-parsimonious tree differed from the one shown only in the arrangement of species in the Halomonas eurihalina-Halomonas elongata-Halomonas halmophila cluster. For abbreviations see the legend to Fig. 1.

comes even less clear. At a level of similarity of $96 \%$ groups 5 and 6 merge and group 6 merges with group $8 . D$. pacifica is equidistant between $D$. cupida and $D$. salina at a level of similarity of $96.5 \%$. However, the level of similarity between $D$. salina and Halomonas eurihalina is $96.7 \%$, but the level of similarity between Halomonas eurihalina and $D$. cupida is only $94.3 \%$.

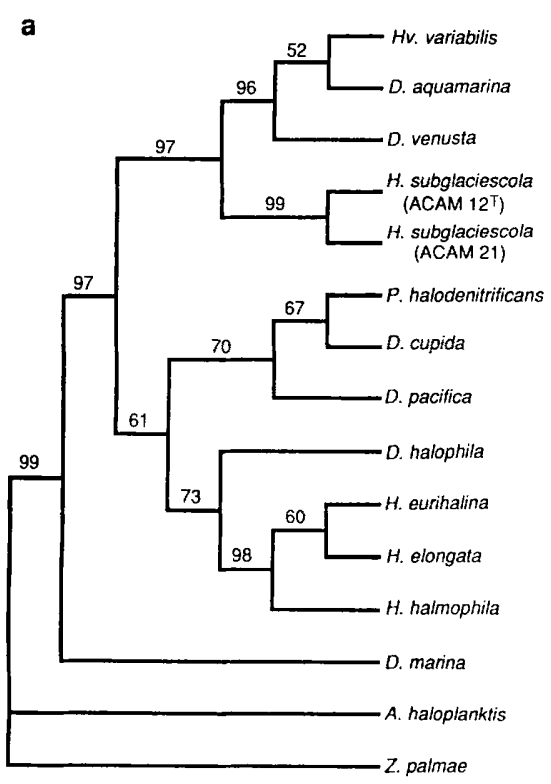

TABLE 2. Frequency of occurrence of signature characteristics of members of the genera Deleya, Halomonas, and Halovibrio and the misnamed organism Paracoccus halodenitrificans in other members of the gamma subclass of the Proteobacteria

\begin{tabular}{|c|c|c|c|}
\hline \multirow{2}{*}{ Position(s) } & \multirow{2}{*}{$\begin{array}{c}\text { Sequence or } \\
\text { nucleotide }\end{array}$} & \multicolumn{2}{|c|}{ Frequency $(\%)$ in ${ }^{a}:$} \\
\hline & & 1995 & 1992 \\
\hline $76-93$ & 6-bp stem & 7.4 & 10.5 \\
\hline 484 & A & 3.2 & 2.6 \\
\hline 486 & $\mathrm{C}$ & 0.5 & 0 \\
\hline 640 & $\mathrm{G}$ & 7.6 & 7.9 \\
\hline $660^{-b}$ & A- & 9.0 & 2.6 \\
\hline 745 & $\mathrm{U}$ & 20.6 & 18.4 \\
\hline $668-$ & A- & 11.3 & 13.2 \\
\hline 738 & $\mathrm{U}$ & 10.6 & 13.2 \\
\hline $669-$ & A- & 13.4 & 15.8 \\
\hline 737 & $\mathrm{U}$ & 13.4 & 15.8 \\
\hline 776 & $\mathrm{U}$ & 11.8 & 2.6 \\
\hline 1124 & $\mathrm{U}$ & 19.2 & 7.9 \\
\hline 1297 & $\mathrm{U}$ & 23.6 & 18.4 \\
\hline 1298 & $\mathrm{C}$ & 3.5 & 10.5 \\
\hline 1423 & A & 3.0 & 10.5 \\
\hline 1424 & $\mathrm{C}$ & 18.2 & 13.2 \\
\hline 1439 & $\mathrm{U}$ & 2.3 & 5.3 \\
\hline 1462 & A & 2.1 & 5.3 \\
\hline 1464 & C & 4.8 & 5.3 \\
\hline
\end{tabular}

${ }^{a}$ In 1995, 433 sequences of members of the gamma subclass of the Proteobacteria (other than members of the genera Deleya, Halomonas, and Halovibrio and the misnamed organism Paracoccus halodenitrificans) were available from the Ribosomal Database Project when the manuscript was prepared. In 1992, 38 such sequences were available.

${ }^{b}$ The dash indicates base pairing with the next nucleotide listed.

When the overall arrangement of species within the Halomonadaceae is considered, it appears that there are three subgroups containing the following organisms: subgroup i, Halomonas halmophila, Halomonas elongata, Halomonas eurihalina, D. salina, D. halophila, D. pacifica, D. cupida, and Paracoccus halodenitrificans; subgroup ii, Halomonas subglaciescola ACAM 21 and ACAM 12 ${ }^{\mathrm{T}}$, Halomonas halodurans, D. venusta, D.

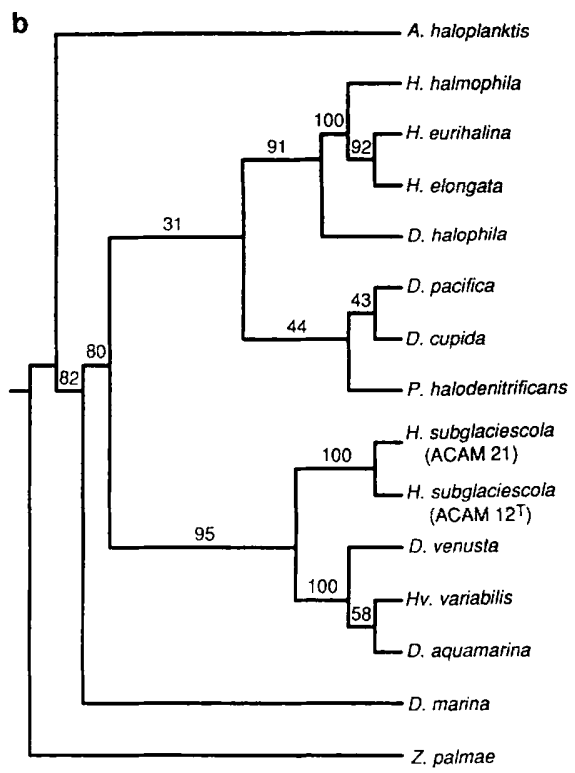

FIG. 3. Bootstrap consensus trees derived by parsimony (a) and distance (b) methods. The numbers shown are the percentages of groups which occurred on bootstrap trees (2,000 replications). For abbreviations see the legend to Fig. 1. 
TABLE 3. Percentages of members of the gamma subclass of the Proteobacteria that contain different numbers of the signature characteristics of the group containing species belonging to the genera Deleya, Halomonas, and Halovibrio and the misnamed organism Paracoccus halodenitrificans

\begin{tabular}{|c|c|}
\hline $\begin{array}{l}\text { No. of signature } \\
\text { characteristics in the } \\
\text { 16S rRNA sequence }\end{array}$ & $\begin{array}{l}\% \text { of members of } \\
\text { the gamma subclass } \\
\text { that contain this } \\
\text { no. of signature } \\
\text { characteristics }\end{array}$ \\
\hline $1 \ldots \ldots \ldots . . . . . .$. & ........... 25 \\
\hline $2 \ldots \ldots \ldots \ldots$ & ........... 21 \\
\hline $3 \ldots \ldots \ldots \ldots$ & …...... 8.3 \\
\hline $4 \ldots \ldots \ldots \ldots \ldots$ & ......... 4.6 \\
\hline $5 \ldots \ldots \ldots \ldots$ & .......... 4.8 \\
\hline $6 \ldots \ldots \ldots \ldots \ldots$ & $\begin{array}{l}\ldots .0 \\
\ldots \ldots \ldots \ldots . . .\end{array}$ \\
\hline $7 \ldots \ldots \ldots$ & $\ldots .9$ \\
\hline $8 \ldots \ldots \ldots \ldots \ldots$ & ......... 1.2 \\
\hline 9 & ............. \\
\hline $10 \ldots \ldots \ldots \ldots \ldots$ & …........ 0.7 \\
\hline $11 \ldots \ldots \ldots \ldots \ldots$ & ........... 0 \\
\hline $12 \ldots \ldots \ldots \ldots \ldots$ & …...... 0 \\
\hline $13 \ldots \ldots \ldots \ldots$ & $\begin{array}{ll}\ldots \ldots \ldots \ldots & 0\end{array}$ \\
\hline $14 \ldots \ldots \ldots \ldots \ldots$ & 0 \\
\hline $15 \ldots \ldots \ldots \ldots$ & ............. \\
\hline $16 \ldots \ldots \ldots \ldots$ & .............. \\
\hline $17 \ldots \ldots \ldots$ & $\begin{array}{l}\ldots \ldots \ldots . . . \\
\ldots . . . . .\end{array}$ \\
\hline $18 \ldots \ldots \ldots \ldots$ & .............. \\
\hline $19 \ldots \ldots \ldots \ldots$ & $\begin{array}{ll}\ldots \ldots \ldots . . . & 0\end{array}$ \\
\hline
\end{tabular}

${ }^{a}$ Excluding members of the genera Deleya, Halomonas, and Halovibrio and the misnamed organism Paracoccus halodenitrificans, all of which contain the 19 signature characteristics.

aquamarina, Halomonas meridiana, and Halovibrio variabilis; and subgroup iii, D. marina. However, in this arrangement there are some species which are as closely related to species belonging to other subgroups as they are to members of their own subgroup. For example, $D$. halophila is as closely related to $D$. marina as it is to $D$. cupida. Levels of similarity for species belonging to subgroup $\mathrm{i}$ are as low as $93.8 \%$, and levels of similarity for species belonging to subgroup ii are as low as $94.4 \%$, whereas some intersubgroup levels of similarity are higher than these values. For example, the levels of similarity between $D$. venusta and $D$. pacifica and between $D$. marina and D. pacifica are 95.0 and $94.9 \%$, respectively. Several groups of species in the bootstrap consensus tree derived from the distance analysis occurred on less than $75 \%$ of the trees, indicating that there was some instability in the arrangement of species in the trees. The lack of resolution of subgroups within this set of species (except at high levels of sequence similarity) is associated with short internodal distances betwen the junction of the three subgroups and the branch points of species within the subgroups (Fig. 1, internodes a through c).

All species belonging to the genera Deleya, Halomonas, and Halovibrio and the misnamed organism Paracoccus halodenitrificans contain the same major fatty acids $(10,30)$. While the proportions of these fatty acids vary among species, the differences among some members of different subgroups are too small to be useful for delineating genera (5). When differences are so small, it is not possible to accurately predict the subgroup to which an unknown organism may belong on the basis of its fatty acid profile. For example, D. cupida and D. pacifica are in different subgroups (level of 16S rRNA sequence similarity; $96.5 \%$ ), and the differences in the proportions of the major fatty acids $16: 0,16: 1$ plus $17 \mathrm{cyc}$, and $18: 1$ plus $19 \mathrm{cyc}$ of these organisms are $3.6,1.9$, and $0.4 \%$, respectively. D. venusta and $D$. aquamarina are in the same subgroup (level of $16 \mathrm{~S}$ rRNA sequence similarity, $98.0 \%$ ), and the differences in the proportions of the major fatty acids $16: 0,16: 1$ plus $17 \mathrm{cyc}$, and $18: 1$ plus 19 cyc are $1.5,2.3$, and $2.1 \%$, respectively. $D$. aquamarina and Halomonas meridiana are members of the same rRNA species complex and exhibit 100\% 16S rRNA sequence similarity; the differences in the proportions of fatty acids 16:0, $16: 1$ plus $17 \mathrm{cyc}$, and $18: 1$ plus $19 \mathrm{cyc}$ in these organisms are similar to the differences in the proportions found in inter- and intrasubgroup comparisons $(1.9,0.7$, and $2.7 \%$, respectively).

The genus Volcaniella was created on the basis of phenotypic characteristics, including morphology, motility, oxidase test results, the ability to produce acid from glucose and other carbohydrates, and the ability to grow on different substrates as sole sources of carbon, nitrogen, and energy (23). The high level of $16 \mathrm{~S}$ rRNA similarity between Halomonas eurihalina and Halomonas elongata (99.1\%), which resulted in the transfer of $V$. eurihalina to the genus Halomonas (18), illustrates that these phenotypic characteristics can no longer be considered appropriate markers to describe genera in this group of organisms. Halovibrio variabilis has a vibroid morphology, and yet it forms a monophyletic group with $D$. aquamarina, Halomonas meridiana, and $D$. venusta. Halovibrio variabilis grows optimally in media containing $9.4 \% \mathrm{NaCl}$, whereas Halomonas meridiana grows optimally in media containing 1 to $3 \% \mathrm{NaCl}$. While many phenotypic characteristics are not useful for describing genera in this group, they are very useful for describing and differentiating species.

The species which currently belong to the genera Deleya, Halomonas, and Halovibrio and the misnamed organism Paracoccus halodenitrificans form a well-defined monophyletic group containing 10 well-resolved subgroups at a level of 16S rRNA sequence similarity of $98.0 \%$; however, resolution of the subgroups is lost at lower levels of similarity. These 10 subgroups cannot be distinguished on the basis of their fatty acid or polar lipid profiles, and all members of all subgroups contain ubiquinone 9 as their major respiratory lipoquinone. The phenotypic characteristics used to define genera within this group of species have consistently failed to delineate phylogenetically meaningful taxa. As we cannot identify chemotaxonomic and phenotypic characteristics that distinguish the genotypic subgroups, these organisms must be included in a single genus.

At some point in the future it might be possible to identify chemotaxonomic or phenotypic characteristics which can be used to differentiate the 10 genotypic subgroups; however, this possibility does not justify perpetuation of the current confusing taxonomy. According to Rule 44 of the International Code of Nomenclature of Bacteria (16), when genera are unified, the genus name used should be the name associated with the type species having the earliest legitimate name. Consequently, we propose that the genera Deleya, Halomonas, and Halovibrio should be united in a single genus, the genus Halomonas, and that Paracoccus halodenitrificans should be transferred to the genus Halomonas. The new name combinations are as follows: Halomonas aquamarina (Deleya aquamarina Akagawa and Yamasato 1989), Halomonas variabilis (Halovibrio varabilis Fendrich 1988), Halomonas venusta (Deleya venusta Baumann et al. 1983), Halomonas halodenitrificans (Paracoccus halodenitrificans Robinson and Gibbons 1952), Halomonas cupida (Deleya cupida Baumann et al. 1983), Halomonas pacifica (Deleya pacifica Baumann et al. 1983), Halomonas marina (Deleya marina Baumann et al. 1983), Halomonas halophila (Deleya halophila Quesada et al. 1984), and Halomonas salina (Deleya salina Valderrama et al. 1991). The species descriptions are unchanged. An emended description of the genus Halomonas is given below. 
A total of 19 signature characteristics were previously associated with the monophyletic group of organisms now proposed as members of the genus Halomonas. The frequency of these signature characteristics among the other members of the gamma subclass of the Proteobacteria is similar to the frequency found when the signatures were identified by using a much smaller database of sequences. It might be possible to create a more stringent set of signature characteristics if the four characteristics which occur most frequently (at positions $745,1124,1297$, and 1424 [E. coli numbering] [Table 2]) were not included. We do not recommend this, however, because at any time during the development of the database the frequency of occurrence of particular signature characteristics will be biased by the inclusion of some taxa for which there are many sequences and other taxa for which there may be a single sequence present.

Mellado et al. (18) determined the $16 \mathrm{~S}$ rRNA sequences of two strains of $C$. marismortui. The levels of $16 \mathrm{~S}$ rRNA sequence similarity between these two strains and the members of the genus Halomonas range from 92.0 to $94.2 \%$ (Table 1). The $\mathrm{G}+\mathrm{C}$ contents of the DNAs of the $C$. marismortui strains were 62 to $65 \mathrm{~mol} \%$, values which are within the range of values for the genus Halomonas. $C$. marismortui contained the 19 16S rRNA signature characteristics associated with the genus Halomonas (18). Mellado et al. (18) concluded that there are enough phenotypic differences between $C$. marismortui and the "Halomonas-Deleya complex" to warrant classification of C. marismortui as a member of a separate genus. The genus Chromohalobacter can be distinguished from the genus Halomonas by the presence of yellow and blue pigments (32). When other phenotypic traits were examined, C. marismortui was quite similar to the members of the genus Halomonas (including members of the genus Deleya) with which it was compared. $C$. marismortui was differentiated from $D$. cupida only by differences in the ability to grow on some substrates as sole sources of carbon, nitrogen, and energy (32). The recently described genus Pseudoalteromonas includes both pigmented and nonpigmented species (12). Halomonas subglaciescola has been observed to form yellow colonies when it is grown on media containing alanine and (in the case of some strains) hydroxy-L-proline (9). A decision concerning whether $C$. marismortui represents a separate genus within the Halomonadaceae should await further data, including the determination of its lipid profile and identification of its major respiratory lipoquinone(s).

The genus Zymobacter, which contains a single species, $Z$. palmae, is the closest relative of the genera Halomonas and Chromohalobacter. The levels of $16 \mathrm{~S}$ rRNA sequence similarity between $Z$. palmae and members of the genus Halomonas range from 89.3 to $92.5 \%$ (average, $90.8 \%$ ). The $16 \mathrm{~S}$ rRNA sequence of $Z$. palmae contains 15 of the 19 signature characteristics which have been associated with the genus Halomonas, including the rare cytosine residue at position $486 . Z$. palmae is fermentative, producing ethanol from sugars, and was isolated from palm sap (21). The $\mathrm{G}+\mathrm{C}$ content of its DNA is $55.8 \mathrm{~mol} \%$, and it contains ubiquinone 9 . The major elements of its fatty acid profile are similar to those of the members of the genus Halomonas; however, the levels of 16:0 in the profiles of the members of the genus Halomonas range from 15.5 to $32.0 \%$, whereas the level in $Z$. palmae is $51 \%$, and $Z$. palmae contains $<0.5 \% 16: 1$, whereas this fatty acid is present at levels ranging from 2.1 to $32.0 \%$ in members of the genus Halomonas. The latter comparison is more distinct if the proportions of $16: 1$ and $17 \mathrm{cyc}$ are added together. Conversion of a monounsaturated fatty acid to its cyclopropane fatty acid derivative is sensitive to various culture conditions. It is thought that adding the two moieties provides a more stable characteristic (14). The fatty acid profile of $Z$. palmae contains $<0.5 \% 16: 1$ plus $17 \mathrm{cyc}$, while the fatty acid profiles of the members of the genus Halomonas contain 7.7 to $43.1 \% 16: 1$ plus $17 \mathrm{cyc}$. While $Z$. palmae was not isolated from a saline environment, it is highly osmotolerant and can grow in the presence of $50 \%$ (wt/vol) maltose (21). The genus Zymobacter is clearly distinct from the genus Halomonas, but the level of similarity between these two genera indicates that they belong to the same family. Therefore, we propose that the genus Zymobacter should be included in the family Halomonadaceae, and the description of the family Halomonadaceae is emended accordingly below. The 15 signature characteristics common to both genera are retained as descriptive characteristics of members of the family Halomonadaceae, and the remaining four signature characteristics are included in the description of the genus Halomonas within the Halomonadaceae.

The closest relative of the genus Halomonas after Z. palmae is $O$. minutulum. The levels of $16 \mathrm{~S}$ rRNA sequence similarity between $O$. minutulum and the members of the genus Halomonas range from 88.8 to $90.6 \%$ (average, $89.4 \%$ ). The level of $16 \mathrm{~S}$ rRNA similarity between $O$. minutulum and $Z$. palmae is $89.8 \%$. O. minutulum contains only 10 of the signature characteristics associated with the Halomonadaceae and does not contain the rare cytosine residue at position 486 . The major respiratory lipoquinone in $O$. minutulum is ubiquinone 8 , and $O$. minutulum contains $3-\mathrm{OH} 14: 1$ in its fatty acid profile, which has not been found in any species belonging to the genus Halomonas or in $Z$. palmae (26). The chemotaxonomic and genotypic differences between $O$. minutulum and the genera Halomonas and Zymobacter indicate that $O$. minutulum should not be transferred to the family Halomonadaceae.

The results of comparisons of levels of 16S rRNA sequence similarity and DNA-DNA hybridization values for some $\mathrm{Fi}$ brobacter strains and some Bacillus strains suggested that it was unlikely that two strains that exhibited less than $99 \% 16 \mathrm{~S}$ rRNA sequence similarity would have a DNA-DNA hybridization value of $70 \%$ or greater in these groups $(2,8)$. Data for members of the Halomonadaceae are limited. The level of $16 \mathrm{~S}$ rRNA similarity between Halomonas eurihalina and Halomonas elongata is $99.1 \%$, and the level of DNA-DNA hybridization of these taxa is $7 \%$ (31). The level of 16S rRNA similarity between Halomonas subglaciescola ACAM 21 and ACAM $12^{\mathrm{T}}$ was $98.2 \%$. On the basis of this value, the phenotypic differences between strain ACAM 21 and Halomonas subglaciescola ACAM $12^{\mathrm{T}}$, and differences in the proportions of the major fatty acids of these organisms, it is doubtful that ACAM 21 is a member of Halomonas subglaciescola. DNA-DNA hybridization data will be required to confirm this hypothesis. The $16 \mathrm{~S}$ rRNA sequences of strain ACAM 21 and Halomonas halodurans were almost identical. In the terminology of Fox et al. (8), these strains belong to the same rRNA species complex or rRNA superspecies. Determining whether strain ACAM 21 is a member of Halomonas halodurans will require determining the level of DNA-DNA hybridization between the two organisms.

Emended description of the genus Halomonas Vreeland, Litchfield, Martin, and Elliot 1980. Gram-negative, straight or curved, rod-shaped cells. Slight or moderate halophiles; optimal growth occurs in the presence of 0.3 to $2.0 \mathrm{M} \mathrm{NaCl}$. May tolerate $\mathrm{NaCl}$ concentrations up to $5.5 \mathrm{M}$. Aerobic, but some strains are capable of anaerobic growth in the presence of nitrate. Motile by means of peritrichous or polar flagella or nonmotile. Chemoorganotrophic. The major respiratory lipoquinone is ubiquinone 9 , and the major fatty acids are 16:1 plus 17:0cyc, 16:0, and 18:1 plus 19:0cyc. The 16S rRNA sequences 
of members of this genus contain four signature characteristics ( $\mathrm{C}$ at position $1424, \mathrm{U}$ at position $1439, \mathrm{~A}$ at position $1462, \mathrm{C}$ at position 1464 [E. coli numbering]) in addition to the 15 signature characteristics associated with the family Halomonadaceae.

Emendation of the description of the family Halomonadaceae Franzmann, Wehmeyer, and Stackebrandt 1988. Gram-negative, straight or curved, rod-shaped cells. Members are either slight or moderate halophiles or osmotolerant, growing in the presence of high concentrations of sugars. Aerobic or facultatively anaerobic. The major respiratory lipoquinone is ubiquinone 9 , and the major fatty acids are 18:1 plus 19:0cyc and 16:0. The family is a member of the gamma subclass of the Proteobacteria, and its members have 15 signature characteristics in their 16S rRNA sequences, including a very rare cytosine residue at position 486 . The 15 signature characteristics are as follows: a 6-bp stem at positions 76 to $93, \mathrm{~A}$ at position $484, \mathrm{C}$ at position $486, \mathrm{G}$ at position $640, \mathrm{~A}$ at position 660 , $\mathrm{A}$ at position $668, \mathrm{~A}$ at position $669, \mathrm{U}$ at position $737, \mathrm{U}$ at position $738, \mathrm{U}$ at position $745, \mathrm{U}$ at position $776, \mathrm{U}$ at position $1124, \mathrm{U}$ at position $1297, \mathrm{C}$ at position 1298, and A at position 1423 (E. coli numbering). The family comprises three genera, Halomonas, Chromohalobacter, and Zymobacter.

\section{ACKNOWLEDGMENT}

This work was supported by funds from the Australian Research Council.

\section{REFERENCES}

1. Akagawa, M., and K. Yamasato. 1989. Synonymy of Alcaligenes aquamarinus, Alcaligenes faecalis subsp. homari, and Deleya aesta: Deleya aquamarina comb. nov. as the type species of the genus Deleya. Int. J. Syst. Bacteriol. 39: $462-466$.

2. Amann, R. I., C. Lin, R. Key, L. Montgomery, and D. Stahl. 1992. Diversity among Fibrobacter isolates: towards a phylogenetic classification. Syst. Appl. Microbiol. 15:23-31.

3. Baumann, L., R. D. Bowditch, and P. Baumann. 1983. Description of Deleya gen. nov. created to accommodate the marine species Alcaligenes aestus, $A$. pacificus, $A$. cupidus, $A$. venustus, and Pseudomonas marina. Int. J. St. Bacteriol. 33:793-802.

4. Dobson, S. J., R. R. Colwell, T. A. McMeekin, and P. D. Franzmann. 1993. Direct sequencing of the polymerase chain reaction-amplified 16S rRNA gene of Flavobacterium gondwanense sp. nov. and Flavobacterium salegens $\mathrm{sp}$. nov., two new species from a hypersaline Antarctic lake. Int. J. Syst. Bacteriol. 43:77-83.

5. Dobson, S. J., T. A. McMeekin, and P. D. Franzmann. 1993. Phylogenetic relationships between some members of the genera Deleya, Halomonas, and Halovibrio. Int. J. Syst. Bacteriol. 43:665-673.

6. Felsenstein, J. 1989. PHYLIP - phylogeny inference package (version 3.2). Cladistics 5:164-166.

7. Fendrich, C. 1988. Halovibrio variabilis gen, nov., Pseudomonas halophila sp. nov. and a new halophilic aerobic coccoid eubacterium from Great Salt Lake, Utah, USA. Syst. Appl. Microbiol. 11:36-43.

8. Fox, G. E., J. D. Wisotzkey, and P. Jurtshuk, Jr. 1992. How close is close: 16S rRNA sequence identity may not be sufficient to guarantee species identity. Int. J. Syst. Bacteriol. 42:166-170.

9. Franzmann, P. D., H. R. Burton, and T. A. McMeekin. 1987. Halomonas subglaciescola, a new species of halotolerant bacteria isolated from Antarctica. Int. J. Syst. Bacteriol. 37:27-34.

10. Franzmann, P. D., and B. J. Tindall. 1990. A chemotaxonomic study of members of the family Halomonadaceae. Syst. Appl. Microbiol. 13:142-147.

11. Franzmann, P. D., U. Wehmeyer, and E. Stackebrandt. 1989. Halomonadaceae fam. nov., a new family of the class Proteobacteria to accommodate the genera Halomonas and Deleya. Syst. Appl. Microbiol. 11:16-19.

12. Gauthier, G., M. Gauthier, and R. Christen. 1995. Phylogenetic analysis of the genera Alteromonas, Shewanella, and Moritella using genes coding for small-subunit rRNA sequences and division of the genus Alteromonas into two genera, Alteromonas (emended) and Pseudoalteromonas gen. nov., and proposal of twelve new species combinations. Int. J. Syst. Bacteriol. 45: $755-761$.
13. James, S. R., S. J. Dobson, P. D. Franzmann, and T. A. McMeekin. 1990. Halomonas meridiana, a new species of extremely halotolerant bacteria isolated from Antarctic saline lakes. Syst. Appl. Microbiol. 13:270-278.

14. Jantzen, E., and K. Bryn. 1985. Whole-cell and lipopolysaccharide fatty acids and sugars of Gram-negative bacteria, p. 145-172. In M. Goodfellow and D. E. Minnikin (ed.), Chemical methods in bacterial systematics. Academic Press, Inc., London.

15. Kersters, K. 1992. The genus Deleya, p. 3189-3197. In A. Balows, H. G. Trüper, M. Dworkin, W. Harder, and K.-H. Schleifer (ed.), The prokaryotes. A handbook on the biology of bacteria: ecophysiology, isolation, identification, applications, 2nd ed., vol. 4. Springer-Verlag, New York.

16. Lapage, S. P., P. H. A. Sneath, E. F. Lessel, V. B. D. Skerman, H. P. R. Seeliger, and W. A. Clarke (ed.). 1992. International code of nomenclature of bacteria. 1990 revision. American Society for Microbiology, Washington, D.C.

17. Larsen, H. 1986. Halophilic and halotolerant microorganisms-an overview and historical perspective. FEMS Microbiol. Rev. 39:3-7.

18. Mellado, E., E. R. B. Moore, J. J. Nieto, and A. Ventosa. 1995. Phylogenetic inferences and taxonomic consequences of $16 \mathrm{~S}$ ribosomal DNA sequence comparison of Chromohalobacter marimortui, Volcaniella eurihalina, and Deleya salina and reclassification of $V$. eurihalina as Halomonas eurihalina comb. nov. Int. J. Syst. Bacteriol. 45:712-716.

19. Miller, J. M., S. J. Dobson, P. D. Franzmann, and T. A. McMeekin. 1994. Reevaluating the classification of Paracoccus halodenitrificans with sequence comparisons of $16 \mathrm{~S}$ ribosomal DNA. Int. J. Syst. Bacteriol. 44:360-361.

20. Murray, R. G. E., D. J. Brenner, R. R. Colwell, P. De Vos, M. Goodfellow, P. A. D. Grimont, N. Pfennig, E. Stackebrandt, and G. A. Zavarin. 1990. Report of the Ad Hoc Committee on Approaches to Taxonomy within the Proteobacteria. Int. J. Syst. Bacteriol. 40:213-215.

21. Okamoto, T., H. Taguchi, K. Nakamura, H. Ikenaga, H. Kuraishi, and K. Yamasato. 1993. Zymobacter palmae gen. nov., sp. nov., a new ethanolfermenting peritrichous bacterium isolated from palm sap. Arch. Microbiol. 160:333-337.

22. Olsen, G. J., N. Larsen, and C. R. Woese. 1991. The Ribosomal RNA Database Project. Nucleic Acids Res. 19(Suppl.):2017-2021.

23. Quesada, E., M. J. Valderrama, V. Bejar, A. Ventosa, M. C. Gutierrez, F. Ruiz-Berraquero, and A. Ramos-Cormenzana. 1990. Volcaniella eurihalina gen. nov., sp. nov., a moderately halophilic nonmotile gram-negative rod. Int. J. Syst. Bacteriol. 40:261-267.

24. Quesada, E., A. Ventosa, F. Ruiz-Berraquero, and A. Ramos-Cormenzana. 1984. Deleya halophila, a new species of moderately halophilic bacteria. Int. J. Syst. Bacteriol. 34:287-292.

25. Robinson, J., and N. E. Gibbons. 1952. The effects of salts on the growth of Micrococcus halodenitrificans n. sp. Can. J. Bot. 30:147-154.

26. Sakane, T., and A. Yokota. 1994. Chemotaxonomic investigation of heterotrophic, aerobic and microaerophilic spirilla, the genera Aquaspirillum, Magnetospirillum and Oceanospirillum. Syst. Appl. Microbiol. 17:128-134.

27. Sambrook, J., E. F. Fritsch, and T. Maniatis. 1989. Molecular cloning: a laboratory manual, 2nd ed. Cold Spring Harbor Laboratory Press, Cold Spring Harbor, N.Y.

28. Stackebrandt, E. 1992. Unifying phylogeny and phenotypic diversity, p. 1947. In A. Balows, H. G. Trüper, M. Dworkin, W. Harder, and K.-H. Schleifer (ed.), The prokaryotes. A handbook on the biology of bacteria: ecophysiology, isolation, identification, applications, 2nd ed., vol. 1. Springer-Verlag, New York.

29. Swofford, D. L. 1990. PAUP: phylogenetic analysis using parsimony, version 3.1. Illinois Natural History Survey, Champaign.

30. Urakami, T., H. Araki, H. Oyanagi, K.-I. Suzuki, and K. Komagata. 1990. Paracoccus aminophilus $\mathrm{sp}$. nov. and Paracoccus aminovorans sp. nov., which utilize $N, N$-dimethylformamide. Int. J. Syst. Bacteriol. 40:287-291.

31. Valderrama, M. J., E. Quesada, V. Bejar, A. Ventosa, M. C. Gutierrez, F. Ruiz-Berraquero, and A. Ramos-Cormenzana. 1991. Deleya salina sp. nov., a moderately halophilic gram-negative bacterium. Int. J. Syst. Bacteriol. 41: 377-384.

32. Ventosa, A., M. C. Gutierrez, M. T. Garcia, and F. Ruiz-Berraquero. 1989. Classification of "Chromobacterium marismortui" in a new genus, Chromohalobacter gen. nov., as Chromohalobacter marismortui comb. nov., nom. rev. Int. J. Syst. Bacteriol. 39:382-386.

33. Vreeland, R. H., C. D. Litchfield, E. L. Martin, and E. Elliot. 1980. Halomonas elongata, a new genus and species of extremely salt-tolerant bacteria. Int. J. Syst. Bacteriol. 30:485-495.

34. Wayne, L. G., D. J. Brenner, R. R. Colwell, P. A. D. Grimont, O. Kandler, M. I. Krichevsky, L. H. Moore, W. E. C. Moore, R. G. E. Murray, E. Stackebrandt, M. P. Starr, and H. G. Trüper. 1987. Report of the Ad Hoc Committee on Reconciliation of Approaches to Bacterial Systematics. Int. J. Syst. Bacteriol. 37:463-464. 